\title{
A VEHICLE ROUTING PROBLEM OPTIMIZATION WITH DRONE USING TABU SEARCH ALGORITHM AND ANALYTICAL HIERARCHY PROCESS
}

\author{
Linda Nuryanti \\ Department of information system, Diponegoro University \\ e-mail: lindnuryanti@gmail.com
}

\begin{abstract}
This research presents optimization of the fastest route using Drone. Vehicle Routing Problem with Drones (VRPD) is used for planning the mapping of areas with minimal makespan. Drone point route design aims to mapping the area with considering the boundaries. In this paper, an optimization method using the Tabu Search algorithm and Analytical Hierarchy Process (AHP) to resolve VRPD problems is proposed. Tabu Search algorithm is suitable for the implementation of Vehicle Routing Problem because it is able to find the closest distance optimally with guiding other processes using a series of movements to change one solution to another. The optimization process using Tabu Search can find a suitable pair of points so that the closest route can be found. AHP is used for the weighting process and determining the hierarchy of route selection by prioritizing routes that meet the criteria and the appropriate weights. The optimization resulted in a $7.08 \%$ reduction in the distance and significantly reduce the makespan as well as the metaheuristic approach. Experimental processes and performance analysis are carried out to find that this method can consistently produce better and optimal solutions.
\end{abstract}

Keywords : Vehicle Routing Problem with Drone; Analytical Hierarchy Process; Tabu Search; Mapping

\section{INTRODUCTION}

The Ministry of Maritime Affairs and Fisheries (KKP) revealed that Indonesia is the largest archipelago in the world, stretching from Sabang to Merauke, Indonesia has 17.499 islands with a total area of approximately 7.81 million $\mathrm{km}^{2}$. Based on the total area, 3.25 million $\mathrm{km}^{2}$ is oceans and 2.55 million $\mathrm{km}^{2}$ is the Exclusive Economic Zone and only about 2.01 million $\mathrm{km}^{2}$ is land. Based on the vast area, Indonesia consists of many islands, a Drone is one of the best solutions that can be used to map the region. Mapping the area using Drone can increase efficiency and reduce risk because it can be controlled remotely (autonomous). Drones have a limited range, where the operation depends on the battery capacity, fuel and transmitter range. Based on these problems, a good mechanism and planning are needed to carry out the mapping so that it can reach all areas optimally.
The shortest route is a search technique to determine the most optimal path. The classic Vehicle Routing Problem (VRP) is used for the delivery and distribution of goods to optimize time and costs [1]. The evolution of Drone technology has opened up many opportunities in transportation and logistics [2], defense, agriculture and public safety. Drones have been successfully applied to the public and private sectors and Drone technology can be used to carry out autonomous flights [3]. In general, there are many assumptions that Drones can move faster between two locations compared to vehicles in general because Drones are not subject to restrictions following road network structures or congestion [4].

Different from the classical VRP, VRPD determine the location to define decisions [5]. The problem becomes more challenging constraint (capacity and distance). Vehicle Routing Problem with Drones (VRPD) is NPhard, the initial solution to this problem can use 
the metaheuristic method [6]. Many effective methods have been developed and are able to finish within a reasonable time, one of which is Tabu Search [7]. In this study, the metaheuristic method is Tabu Search algorithm, it is used to optimize the search for the best Drone route on the mapping plan. Tabu Search algorithm has been proven from various studies related to computing as a simple but effective optimization technique and it has advantages in terms of search, compared to other optimization techniques [1]. The main principle of Tabu Search algorithm is flexible attribute-based memory structure, the evaluation and search criteria are carried out thoroughly. Tabu Search mechanism is carried out by evaluating movements based on the criteria. It uses memory functions based on different time ranges (short to long term) in implementing strategies to focus on specific areas, encourage the search for new territories.

The shortest route planning optimization process has been produced by Tabu Search; it will be combined with the Analytical Hierarchy Process (AHP) to further optimize route determination based on the weighting of the required criteria. The concept of AHP method is a pairwise comparison of the importance of objects or variables at a certain hierarchical level to the next high level [8]. The advantage of AHP is that there is a linguistic scale in the weighting of its criteria [9]. Decision making mechanism with priority ranking based on Drone distance and capacity criteria. The existing criteria will be compared in pairs to produce the best route in the mapping plan.

This paper combines the implementation of Tabu Search algorithm and AHP for mapping planning. Determination of the fastest Drone route is done by finding the problem model and the appropriate algorithm in solving VRPD problems. The implementation of VRPD problem considers the distance between one point and another to determine a more optimal distance. The problem model is made in a conceptual model that represents a system based on the method to help simulate the subject. The planning process is carried out to simplify Drone operation and adjust area coverage based on Drone specifications. The implementation of the Tabu Search algorithm and AHP can produce a more efficient route, that it can assist in mapping plan.

\section{METHODS}

\section{VRPD Model}

VRPD model was first studied to minimize Drone time serving all customers [7]. The formulation of a model in this problem aims to minimize Drone route planning in relation to the total travel time and it used for determining the fastest route of the Drone from one point to another. Drone is use to carry out mapping based on point $T=\{0,1,2, \ldots, n\}$. Drones start their mission from point 0 and return to the original place after completing the mission. VRP involves determining a series of routes that minimize the total transport time. The goal of VRPD is to find a route that allows it to pass through all points with minimal total time. The basic problem in VRP is modelling the problem in a mathematical model and the application of heuristics algorithms to calculate the shortest path. VRP mathematical formulations used to minimize [10]:

$\mathrm{z}=\sum_{d \in U} \sum_{i \in N} \sum_{d \in N} c_{i j} x_{i j d}$

$x_{i j d}=\left\{\begin{array}{lc}1, & \text { if Drone } d \text { passes through point } i, \text { then to point } j \\ 0, & \text { next }\end{array}\right.$

\section{Constraints:}

1. Each point is visited exactly once by one Drone.

$\sum_{d \in U} \sum_{i \in N} \sum_{d \in N} x_{i j d}$

2. Total requests for points visited in one route do not exceed the Drone capacity.

$\sum_{i \in x} d_{i} \sum_{i \in N} x_{i j d} \leq q, \forall d \in V$

3. Each route starting from depot.

$\sum_{j \in N} x_{0 j d}=1, \forall d \in V$

4. Every Drone that has visited a point, it would leave these points.

$\sum_{i \in N} x_{i h d}-\sum_{j \in N} x_{h j d}=0, \forall h \in C, \forall d \in V$

5. Each route ends at $n+1$ depot.

$$
\sum_{i \in N} x_{i, n+1, d}=1, \forall d \in V
$$

6. Change $x_{i j d}$ is a binary.

$$
x_{i j d} \in\{0,1\}, \forall i, j \in N, \forall d \in V
$$

7. Flight path and depot location

$$
\sqrt{\left(X_{a}-X_{b}\right)^{2}+\left(Y_{a}-Y_{b}\right)^{2}}
$$


Table 1. Mathematic Notation.

\begin{tabular}{ll}
\hline Notation & \multicolumn{1}{c}{ Detail } \\
\hline$V$ & $\begin{array}{l}\text { set of vehicles with the same } \\
\text { capacity }\end{array}$ \\
$C$ & Set of flight plan $=\{1, \ldots, n\}$ \\
$A$ & Set of waypoint $=\{(i, j) \mid i, j \in N, i$ \\
& $\neq j\}$ \\
$c_{i j}$ & Distance from vertex $i$ to vertex $j$ \\
$q$ & Drone capacity \\
$X_{a}$ & Starting point $\mathrm{X}$ \\
$X_{b}$ & Destination point $\mathrm{X}$ \\
$Y_{a}$ & Starting point $\mathrm{Y}$ \\
$Y_{b}$ & Destination point $\mathrm{Y}$ \\
\hline
\end{tabular}

Tabu Search Algorithm

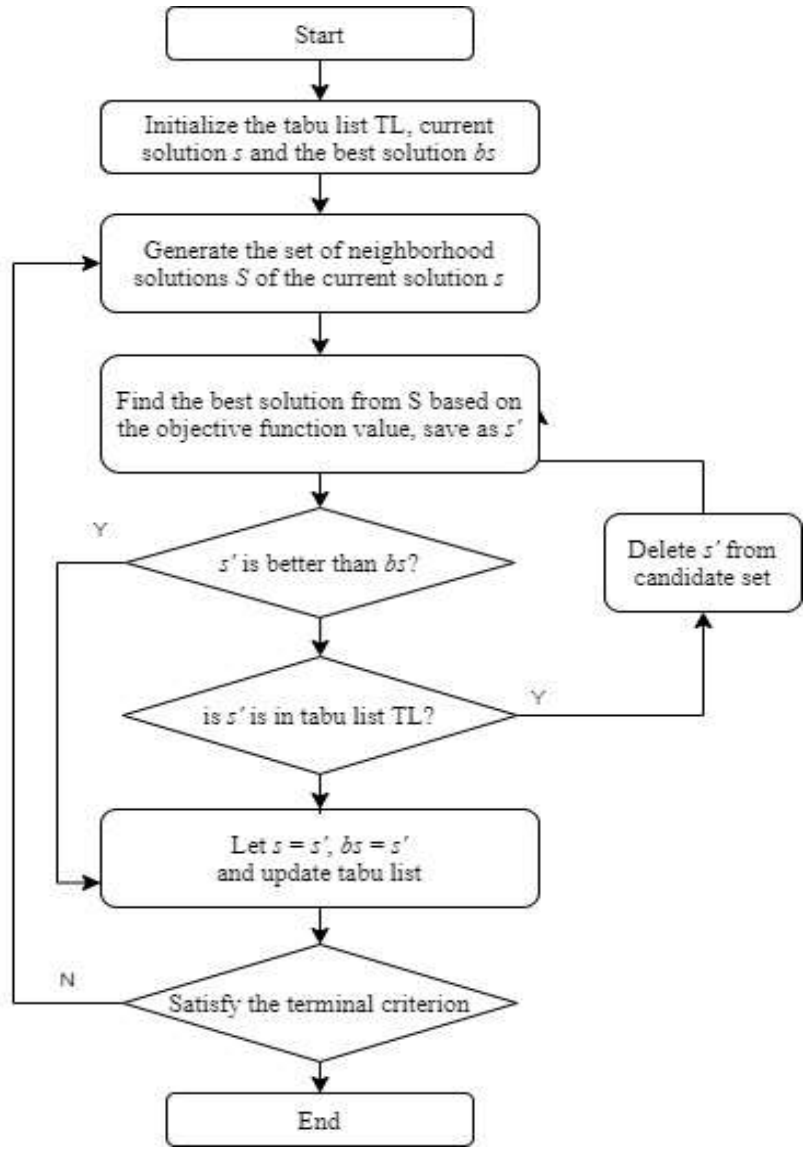

Figure 1. Flowchart Tabu Search.

Tabu Search algorithm are designed to cross the boundaries of feasibility or local optimization and systematically to allow exploration in restricted areas. Tabu Search algorithm has some dependence on the initial solution to find a good final solution [1]. In general, when Tabu Search solving a specific problem, it can be used to generate the best initial solution. Tabu Search is a metaheuristic method that guides local search procedures to explore solution spaces beyond local optimality. Figure 1. is an optimization process procedure on Tabu Search [11].

1. Initial Solution

The initial solution is the first step taken in the Tabu Search Algorithm process, where the initial solution can be formed randomly or a heuristic method that will improve the solution in the next iteration. The initial solution $s=b s$ can be anything according to the criteria for an acceptable solution. The random method will form a route from the start point to the end point without considering the minimum travel time for each point.

2. Define Tabu Search component

After forming the initial solution, the process of determining several control parameters, such as the tabu tenure, tabu list length and the number of candidate solutions that will be calculated.

3. Find the neighbourhood solution

The neighbourhood solution is an alternative solution obtained by moving nodes, it explores the solution by replacing recent solution. This step result in a set of solutions $s$ become a new neighbourhood solution $S$. Based on the set of solutions, the existing solutions in the tabu list will be deleted except those that match the aspiration criteria and the new set of results $(S)$. The process usage of flexible memory and exploits the earlier state of search.

$s \in S=\{S-T L\}+A$

4. Find the best solution

The process of finding the best solution using tabu list. Tabu list contains the move attribute that has been found previously, which looks for a new solution based on the previous solution $(S)$ and it save as the newest solution $s^{\prime}$. If the $s^{\prime}$ solution is better than the initial solution (bs), then tabu list update process will be continued in the termination criteria process. 
5. Termination Criteria

Termination criteria is used after all predetermined iterations have been fulfilled. Termination criteria depend on the problem but some possible examples include the maximum number of iterations, if the best solution found is better than some threshold.

Table 2. Dataset Waypoint.

\begin{tabular}{ccc}
\hline Waypoint & Lat & Long \\
\hline 0 & 5.5012256 & 95.6561723 \\
1 & 5.4967285 & 95.6505234 \\
2 & 5.4609873 & 95.6056351 \\
3 & 5.4564893 & 95.5999869 \\
4 & 5.4519269 & 95.6035980 \\
5 & 5.4564248 & 95.6092462 \\
6 & 5.4749000 & 95.6699000 \\
7 & 5.4968776 & 95.6600525 \\
8 & 5.4925295 & 95.6639326 \\
9 & 5.4880324 & 95.6582838 \\
\hline
\end{tabular}

Table 2. is the waypoint dataset used in the Tabu Search process. The data will then be optimized using Tabu Search and then will produce a waypoint representation element data based on the constraints that have been previously set.

\section{Analytical Hierarchy Process (AHP)}

AHP is a Multi Criteria Decision Making (MCDM) method which is used as an efficient method to solve several analytic hierarchical problems [12]. AHP provides an assessment of both quantitative and qualitative criteria based on a pairwise comparison matrix of criteria and alternatives [9]. AHP concept is for analytical decision making which allows the decision maker to choose the best option among a number of alternatives based on several criteria. This process includes assigning attributes to each criterion, integrating qualitative and quantitative analysis and strong systematization [13]. In this study, AHP is used to determine the waypoint representation that will be used for the route selection process using the Tabu Search method.

1. Sum up the value in every column of the matrix pairs.

$$
\mathrm{c}_{i j}=\sum_{i=1}^{n} \mathrm{c}_{i j}
$$

2. Divide every element in the matrix with the column in which the total a normalized matrix.

$$
x_{i j}=\frac{c_{i j}}{\sum_{n-1}^{n} c_{i j}}\left[\begin{array}{lll}
X_{11} & X_{12} & X_{13} \\
X_{21} & X_{22} & X_{23} \\
X_{31} & X_{32} & X_{33}
\end{array}\right]
$$

3. Divide the number of normalized matrix column with the number of criteria used to yield weighted matrix $(n)$.

$$
x W_{i j}=\frac{\sum_{j=1}^{n} x_{i j}}{n}\left[\begin{array}{l}
W_{11} \\
W_{12} \\
W_{13}
\end{array}\right]
$$

This formula used to determine the priority value based on the expert, the assessment from expert will be calculated in AHP method. There are 2 criteria based on finding the nearest route can be seen in Table 3.

Table 3. The criteria to define waypoint representation.

\begin{tabular}{ccc}
\hline Code & Criteria & Weight Value \\
\hline C1 & Distance & Very Near, Near, Far \\
C2 & Capacity & Lots, Enough, Less \\
\hline
\end{tabular}

Table 3. represents the details of criteria used to define the best route. The results of the calculation of criteria will be used to determine the main value that will be used to produce the comparison matrix in order to produce the eigenvalues $\left(W_{i j}\right)$.

\section{RESULTS AND DISCUSSION}

\section{Research Results}

Based on the process that has been carried out, the resulting output is a waypoint representation, the minimum total distance and the route taken by the Drone.

Table 4. Calculation Result of Representation Waypoint.

\begin{tabular}{cccc}
\hline Waypoint & Lat & Long & $\boldsymbol{X}_{\boldsymbol{b}}$ \\
\hline 0 & 5.5012256 & 95.6561723 & - \\
1 & 5.4967285 & 95.6505234 & 1 \\
2 & 5.4609873 & 95.6056351 & 2 \\
3 & 5.4564893 & 95.5999869 & 3 \\
4 & 5.4519269 & 95.6035980 & 4
\end{tabular}




\begin{tabular}{llll}
5 & 5.4564248 & 95.6092462 & 5 \\
6 & 5.4749000 & 95.6699000 & 6 \\
7 & 5.4968776 & 95.6600525 & 9 \\
8 & 5.4925295 & 95.6639326 & 8 \\
9 & 5.4880324 & 95.6582838 & 7 \\
\hline
\end{tabular}

Table 4 . is the calculation result of the waypoint representation based on the optimization process using Tabu Search algorithm. The results of these calculations are used as the basis for calculating the distance between points which can then be used to calculate the total distance.

Table 5. Calculation of Total Distance.

\begin{tabular}{ccc}
\hline $\boldsymbol{X}_{\boldsymbol{a}}$ & $\boldsymbol{X}_{\boldsymbol{b}}$ & Distance \\
\hline 0 & 1 & 0.007220386 \\
1 & 2 & 0.057379377 \\
2 & 3 & 0.007220399 \\
3 & 4 & 0.005818551 \\
4 & 5 & 0.007220337 \\
5 & 6 & 0.063405177 \\
6 & 9 & 0.017532713 \\
9 & 8 & 0.007220308 \\
8 & 7 & 0.00582762 \\
7 & 0 & 0.005827612 \\
\hline
\end{tabular}

Table 5. is the result of the distance calculation based on the equation (8) for the location between the points that the Drone has passed and the result of the total distance calculation. The result of the criteria on Table 3. will be used to create equation comparison matrix.

Table 6. Normalization Matrix of TS Value, Result Data and Eigen Value.

\begin{tabular}{ccc}
\hline Code & C1 & C2 \\
\hline Waypoint 1 & 0.026797722 & 0.013198878 \\
Waypoint 2 & 0.212957672 & 0.104889599 \\
Waypoint 3 & 0.02679777 & 0.013198902 \\
Waypoint 4 & 0.26832134 & 0.132158272 \\
Waypoint 5 & 0.065070866 & 0.032049829 \\
Waypoint 6 & 0.026797432 & 0.013198735 \\
Waypoint 7 & 0.021628614 & 0.010652899 \\
Waypoint 8 & 0.021628584 & 0.010652885 \\
\hline
\end{tabular}

Table 6 is the result of normalization and Eigen Value based on the waypoint results generated on the Tabu Search. The next stage is to connect the results of the optimization of the shortest route that will be traversed by the Drone in the mapping process. The representation of the results of the shortest Drone route can be seen in Figure 2.

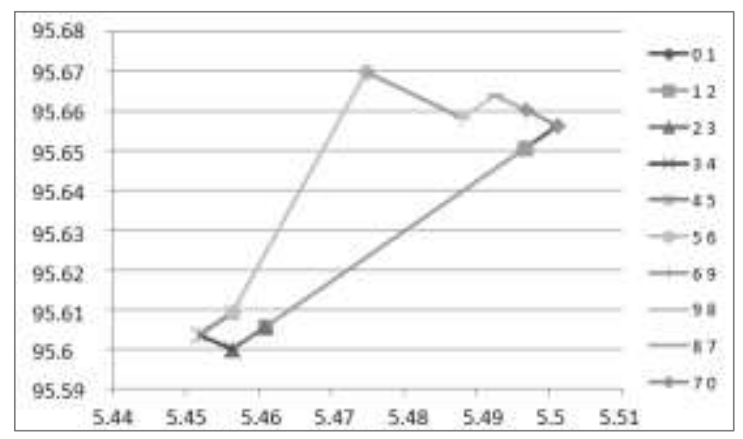

Figure 2. Calculation Result of Waypoint.

Figure 2. is a recommendation waypoint result based on the Tabu Search optimization process and matching criteria using AHP. These results are representation of the sample waypoint data. The results of the search for the shortest route start at depot 0 and return to depot 0 , the results for the shortest route 0-1-2-3-4-5-6-9-8-7-0.

\section{Discussions}

The results of Tabu Search and AHP calculations show the shortest route generated based on the latitude and longitude data sample. The optimization process using Tabu Search is used to perform neighbourhood matching based on VRPD problems by considering the predefined boundaries. The optimization process produces route recommendations that will be used for mapping planning using Drones. AHP method is used for the weighting process and determining the hierarchy of route selection based on predefined parameters. The proposed approach can be used to analyse multi-criteria decision making by prioritizing routes that meet the criteria and the appropriate weights based on latitude and longitude data in Table 2.

The results of the optimization route are compared with Google Maps. The optimization results using Tabu Search and AHP produce the shortest route 0-1-2-3-4-5-6-9-8-7-0 with a total distance $184.67 \mathrm{~km}$ and the total distance before optimization is $198.75 \mathrm{~km}$. The optimization resulted in a $7.08 \%$ reduction in the distance. 
This shows that the planning carried out using the Tabu Search algorithm and AHP can carry out the optimization process by considering Drone limitations. The implementation of these two methods can show the shortest route recommendation in mapping planning using Drones.

\section{CONCLUSION}

In this paper, Tabu Search algorithm is proposed to perform the route optimization process based on AHP alternative ranking. The results of the research to determine the shortest route Drone implemented in a route based on the waypoints that have been carried out by the normalization process. Tabu Search and AHP are easy to implement in VRPD implementation because they can provide the best alternative in solving complex decision problems. In further research, it is necessary to combine other optimization methods on the various dataset waypoint in order to produce a more optimal solution and use real data for more valid result.

\section{AUTHOR INFORMATION}

\section{Corresponding Authors}

Email: lindnuryanti@gmail.com. Phone: +62 81280771683

\section{Author Contributions}

First Author have fully contributed to this work.

\section{ACKNOWLEDGMENTS}

We would like to thank the reviewers for carefully reading our manuscript and for giving such constructive, insightful comments and suggestions, which substantially helped to improve the quality of our paper.

\section{REFERENCES}

[1] M. Qiu, Z. Fu, R. Eglese, and T. Qiong. "A Tabu Search algorithm for the vehicle routing problem with discrete split deliveries and pickups", Computers and operations research, 2018, Vol.100, pp.102-116.

[2] A. Karak, and K. Abdelghany. "The hybrid vehicle-drone routing problem for pick-up and delivery services", Transportation Research Part C, 2019, Vol.102, pp.427449.

[3] A. Otto, N. Agatz, J. Campbell, B. Golden, and E. Pesch. "Optimization approaches for civil applications of unmanned aerial vehicles (UAVs) or aerial Drones: A survey", Networks, 2018, pp.1-48.

[4] D. Schermer, M. Moeini, and O. Wendt. "A matheuristic for the vehicle routing problem with Drones and its variants", Transportation Research Part C, 2019, Vol.106, pp.166-204.

[5] Z. Wang, and J. B. Sheu. "Vehicle routing problem with Drones", Transportation Research Part B, 2019, Vol.122, pp.350364.

[6] D. Schermer, M. Moeini, and O. Wendt. "A hybrid VNS/Tabu Search algorithm for solving the vehicle routing problem with Drones and en route operations", Computers and Operations Research, 2019, Vol.109, pp.134-158.

[7] J. Euchi, and A. Sadok. "Hybrid geneticsweep algorithm to solve the vehicle routing problem with Drones", Physical Communication, 2021, Vol.44, Article 101236.

[8] O. Hoscan, and S. Cetinyokus. "Determination of emergency assembly point for industrial accidents with AHP analysis", Journal of Loss Prevention in the Process Industries, 2021, Vol.69, Article 104386.

[9] J. Fang, and F. Y. Partovi. "Criteria determination of analytic hierarchy process using a topic model", Expert Systems with Applications, 2021, Vol.169, Article 114306.

[10] B. Kallehauge, J. Larsen, O. B. G, Madsen and M. M. Solomon. "Vehicle routing problem with time windows", Column Generation, 2002.

[11] J. Peng, H. Li, Q. Jiang, Y. Wang, and J. Chen. "An integrative approach for measuring semantic similarities using gene ontology", BMC Systems Biology, 2014.

[12] T. Aykut. "Determination of groundwater potential zones using Geographical Information Systems (GIS) and Analytic Hierarchy Process (AHP) between EdirneKalkansogut (northwestern Turkey)", Groundwater for Sustainable Development, 2021, Vol.12, Article 100545.

[13] G. Li, and Y. Li. "Optimization spatial pattern method for vegetation landscape in bay based on AHP", Microprocessors and Microsystems, 2021, Vol.83, Article 104041. 